\title{
Influência do substrato sobre o crescimento de Subulina octona (Brugüière) (Mollusca, Subulinidae), sob condições de laboratório
}

\author{
Sthefane D'ávila \& Elisabeth Cristina de Almeida Bessa
}

Departamento de Zoologia, Instituto de Ciências Biológicas, Universidade Federal de Juiz de Fora. Campus Universitário, 36036-330 Juiz de Fora, Minas Gerais, Brasil. E-mail: sthefanedavila@hotmail.com

\begin{abstract}
Influence of substrate on growth of Subulina octona (Brugüière) (Mollusca, Subulinidae), under laboratorial conditions. The influence of substrate on growth of Subulina octona (Brugüière, 1789), reared in sand, clay and humus, was investigated under laboratorial conditions. The individuals reared in humus, showed smaller shell lengths at the ages of 15 days and 30 days, and at the first, second and third reproductive events. There was a progressive decline in growth rate of shell length with time. This fact was observed for the individuals kept in the three kinds of substrate, demonstrating that the indeterminate growth, with a progressive decline in growth after maturity, is the strategy exhibited by S. octona. In this study was also observed that S. octona of different developmental stages fed on substrate and the molluscs reared in humus consumed less food. This is probably due to the fact that humus is more likely to be take as food resource. So that, the snails reared in humus tend to consume more substrate and less ration, obtaining less proteins, carbohydrates and calcium than those snails reared in sand and clay. This low intake of ration might have negative effects over the growth of the snails.
\end{abstract}

KEY WORDS. Land snail, life cycle, substrate.

RESUMO. Os moluscos pulmonados selecionam substratos adequados a sua sobrevivência, exibindo preferência por determinadas características químicas, físicas e estruturais. Esse fator ambiental afeta a reprodução e a sobrevivência desses animais. Todavia, ainda permanece obscuro que aspectos do ciclo de vida e comportamento são influenciados. $O$ presente trabalho teve por objetivo determinar se o substrato influencia o crescimento de indivíduos da espécie Subulina octona (Brugüière, 1789), mantidos em areia, argila e terra vegetal. Indivíduos mantidos em caixa com terra vegetal, apresentaram menor comprimento de concha aos 15 e 30 dias de vida, no primeiro, segundo e terceiro evento reprodutivo, quando comparados a indivíduos criados em caixas com areia e argila. Foi observada uma tendência para a desaceleração do crescimento dos indivíduos submetidos aos três tratamentos, mostrando que o crescimento indeterminado, com o declínio progressivo do crescimento após a maturidade, é a estratégia exibida por S. octona. No presente estudo, foi observado que $S$. octona ingeria substrato regularmente e que os indivíduos criados em terra vegetal consumiam menos ração. É provável que a terra vegetal forneça condições semelhantes ao habitat preferencial dos moluscos em ambiente natural e seja mais propícia à utilização como recurso alimentar. Dessa forma, os moluscos criados em terra vegetal tenderam a consumir mais substrato e menos ração do que aqueles criados em areia e argila, obtendo quantidade menor de nutrientes, o que pode ter influenciado seu crescimento.

PALAVRAS CHAVE. Ciclo de vida, ingestão de substrato, moluscos terrestres.

Dentre os moluscos pulmonados com importância médicoveterinária, destaca-se a espécie Subulina octona (Brugüière, 1789) (Subulinidae), a qual participa como hospedeiro intermediário nos ciclos de Platynosomum illiciens (Braum, 1901) (Dicrocellidae), Postharmostomum gallinum Witenberg, 1923 (Brachylaimidae), Tanaisia (Paratanaisia) bragai (Santos, 1934) (Dicrocellidae), Aerulostrongylus abstrusus (Railliet, 1898) (Angiostrongylidae), Angiostrongylus vasorum (Baillet) (Angiostrongylidae) e Davainea proglottina (Davaine, 1860) (Davaneidae) (ARAúJo \& BESSA 1993).
O primeiro registro desta espécie no Brasil foi feito por Fisher \& Crosse (1878), para os estados do Ceará e Bahia. Atualmente, S. octona apresenta ampla distribuição geográfica, devido principalmente à introdução por meio de comércio de vegetais, sendo relatada sua ocorrência no Brasil, nos estados do Amapá, Pará, Bahia, Rio de janeiro, Rio grande do Sul, Minas Gerais, São Paulo, Paraná, Amazonas e Rondônia; bem como em quase todo o continente americano, África, Europa, Austrália e Índias Orientais (ARAÚJo \& BESSA 1993).

Para que formas eficientes de manejo, controle e conser-

Revista Brasileira de Zoologia 22 (1): 205-211, março 2005 
vação dessa espécie sejam executadas, mostra-se necessário conhecer os diversos aspectos relacionados ao seu comportamento e bioecologia.

Dentre os fatores abióticos que afetam o ciclo de vida dos moluscos pulmonados, o substrato apresenta grande importância por constituir uma fonte de umidade, alimento, proteção mecânica, camuflagem e sítios para oviposição, afetando o crescimento, reprodução, sobrevivência e distribuição desses animais (Elwell \& Ulmer 1971, Clampit 1973, Nihei et al. 1981, OuTEIRO et al. 1989, ONDina et al. 1998, CoOK 1985, Voss et al. 2001).

Teve-se por objetivo determinar se o substrato influencia o crescimento de indivíduos da espécie $S$. octona. Para tanto, foram analisados os seguintes parâmetros: crescimento desde o nascimento, até o alcance da maturidade sexual e o comprimento da concha dos indivíduos a cada evento reprodutivo.

\section{MATERIAL E MÉTODOS}

A metodologia utilizada para a obtenção e manutenção dos moluscos, assim como a formação dos grupos experimentais e os tratamentos empregados foi idêntica à descrita por D’Ávila E BESSA (2005), em um estudo sobre a influência do substrato sobre a reprodução de $S$. octona.

As observações foram realizadas durante 120 dias, de 22 de novembro de 2001 a 21 de março de 2002 . O crescimento de 115 indivíduos, mantidos em caixa com areia (caixa1, com 35 indivíduos), caixa com argila (caixa 2, com 35 indivíduos) e caixa com terra vegetal (caixa 3 , com 35 indivíduos), foi avaliado por meio de medições quinzenais (até o $30^{\circ}$ dia de vida), com o auxílio de um paquímetro Kanon (Mardened Stainless 1/28 in 1/20 mm).

Foram realizadas observações diárias, obtendo-se, para todos os moluscos de cada caixa, o comprimento da concha no início do experimento, aos 15 e 30 dias de vida; o comprimento da concha a cada evento reprodutivo e o aumento do comprimento da concha, de um evento reprodutivo para o subseqüente. Para a análise estatística dos dados foi utilizado o teste de análise de variância (One way ANOVA), com intervalo de confiança de 95\%, seguido pelo teste de Scheffe ou Tamhane. Para a verificação da existência de correlação entre variáveis utilizou-se o teste de Spearman.

Para verificar a existência de correlação entre o peso, o comprimento da concha e o número de ovos produzidos por S. octona, foram utilizados 60 indivíduos, de diferentes idades, provenientes das criações matrizes do Laboratório de Moluscos, Universidade Federal de Juiz de Fora. Os moluscos foram pesados em uma balança de precisão, o comprimento da concha foi medido com o auxílio de um paquímetro e os ovos, visíveis por transparência da concha, contados. Sobre os dados obtidos foi realizado um teste de correlação (Teste de correlação de Spearman, $\mathrm{p}<0,01$ ).

\section{RESULTADOS E DISCUSSÃO}

O substrato influenciou o crescimento de S. octona. Indivíduos mantidos em caixa com terra vegetal apresentaram menor comprimento de concha aos 15 e 30 dias de vida, no primeiro, segundo e terceiro evento reprodutivo, quando comparados a indivíduos criados em caixas com areia e argila. Observa-se na tabela I as medidas de comprimento da concha dos indivíduos das caixas 1 (areia), 2 (argila) e 3 (terra vegetal) recém eclodidos, com 15 e 30 dias. A análise de variância (ANOVA, $\mathrm{p}<0,05)$ mostrou ser significativa a diferença entre as médias do comprimento das conchas, aos 15 dias de vida (Tab. I), dos indivíduos da caixa 1 e 3 (diferença entre as médias: 3,0009, sig.: 0,000); bem como entre os indivíduos da caixa 2 e 3 (terra vegetal) (diferença entre as médias: 3,1303, sig.:0,000).

A média do comprimento das conchas dos indivíduos da caixa 3, aos 30 dias de vida (Tab. I) foi significativamente mais baixa que a média do comprimento das conchas dos indivíduos das caixas 1 e 2, sendo a diferença entre as médias 2,7689 e 2,9214 e a significância, 0,000 e 0,000, respectivamente.

No presente estudo, foi observado que $S$. octona ingeria substrato regularmente e que os indivíduos criados em terra vegetal consumiam menos ração. É provável que a terra vegetal forneça condições semelhantes ao habitat preferencial dos moluscos em ambiente natural (DuTRA 1980) e seja mais propícia à utilização como recurso alimentar (CHATFIELD 1976, AsAMI \& Obayashi 1999, Chevalier et al. 2001, Speiser 2001). Dessa forma, os moluscos criados em terra vegetal tenderam a consumir mais substrato e menos ração do que aqueles criados em areia e argila, obtendo quantidade menor de nutrientes (MANsur \& Machado 1994, Bessa \& Araújo 1995c), o que pode ter influenciado seu crescimento.

\section{Comprimento da concha por evento reprodutivo}

A tabela II traz as medidas do comprimento da concha, a cada evento reprodutivo dos indivíduos da caixa 1 (areia), 2 (argila) e 3 (terra vegetal), respectivamente. O teste de correlação de Spearman foi utilizado para se verificar a existência de correlação entre o comprimento da concha em cada evento reprodutivo e no evento subseqüente. Os resultados obtidos para os moluscos de cada substrato são apresentados separadamente e, em seguida, analisados comparativamente.

Caixa 1 (areia): houve correlação entre o comprimento da concha, no primeiro e segundo evento ( $\mathrm{p}<0,05$; coeficiente de correlação: 0,4332 ; sig.: 0,011 ), no segundo e terceiro evento ( $p<0,01$; coeficiente de correlação: 0,641; sig.: 0,000) e no terceiro e quarto evento ( $\mathrm{p}<0,01$; coeficiente de correlação: 0,933; sig.: 0,000).

Caixa 2 (argila): houve correlação entre o comprimento da concha dos indivíduos no segundo e no terceiro evento $(\mathrm{p}<$ 0,01 ; coeficiente de correlação: 0,810; sig.: 0,000), e entre o comprimento da concha no terceiro e no quarto evento ( $\mathrm{p}<$ 0,05; coeficiente de correlação: 0,596; sig.: 0,015).

Caixa 3 (Terra vegetal): houve correlação entre o comprimento da concha dos indivíduos no primeiro e segundo evento ( $p<0,01$; coeficiente de correlação: 0,738; sig.: 0,000), no segundo e terceiro evento ( $\mathrm{p}<0,01$; coeficiente de correlação: 0,890 ; sig.:0,000) e no terceiro e quarto evento ( $\mathrm{p}<0,01$; coeficiente de correlação: 0,830; sig.: 0,000). 
Tabela I. Comprimento da concha $(\mathrm{mm})$ de indivíduos deSubulina octona, recém-eclodidos, aos 15 e 30 dias de vida, mantidos em diferentes substratos.

\begin{tabular}{|c|c|c|c|c|c|}
\hline Caixas & Mínimo & Máximo & Média & Desvio padrão & Coeficiente de variação (\%) \\
\hline \multicolumn{6}{|l|}{ Recém-eclodidos } \\
\hline Caixa 1 (areia) & 1,5 & 3,0 & 2,41 & 0,50 & 21,00 \\
\hline Caixa 2 (argila) & 1,0 & 3,0 & 2,12 & 0,40 & 19,18 \\
\hline Caixa 3 (terra vegetal) & 1,0 & 3,0 & 2,07 & 0,62 & 29,93 \\
\hline \multicolumn{6}{|l|}{ Aos 15 dias de vida } \\
\hline Caixa 1 (areia) & 4,0 & 8,5 & 6,47 & 1,11 & 17,21 \\
\hline Caixa 2 (argila) & 5,0 & 8,0 & 6,60 & 0,83 & 12,70 \\
\hline Caixa 3 (terra vegetal) & 2,0 & 6,0 & 3,46 & 1,19 & 34,35 \\
\hline \multicolumn{6}{|l|}{ Aos 30 dias de vida } \\
\hline Caixa 1 (areia) & 8,0 & 13,0 & 10,66 & 1,26 & 11,87 \\
\hline Caixa 2 (argila) & 9,0 & 12,5 & 10,81 & 0,95 & 8,83 \\
\hline Caixa 3 (terra vegetal) & 4,0 & 11,0 & 7,89 & 1,58 & 20,13 \\
\hline
\end{tabular}

Tabela II. Comprimento da concha $(\mathrm{mm})$ em cada evento reprodutivo de indivíduos de Subulina octona, mantidos em caixa com areia, argila e terra vegetal por 120 dias.

\begin{tabular}{|c|c|c|c|c|c|}
\hline & Mínimo & Máximo & Média & Desvio padrão & Coeficiente de variação (\%) \\
\hline \multicolumn{6}{|l|}{ Caixa 1 (areia) } \\
\hline Comprimento da concha no $1^{\circ}$ o evento & 13 & 16,0 & 14,08 & 0,90 & 6,380 \\
\hline Comprimento da concha no $2^{\circ}$ evento & 14 & 21,0 & 17,36 & 1,49 & 8,600 \\
\hline Comprimento da concha no 3 o evento & 18 & 22,5 & 20,13 & 1,25 & 6,250 \\
\hline Comprimento da concha no $4^{\circ}$ evento & 19 & 23,0 & 20,81 & 1,21 & 5,840 \\
\hline Comprimento da concha no 5o evento) & 20 & 23,0 & 21,46 & 0,97 & 4,510 \\
\hline \multicolumn{6}{|l|}{ Caixa 2 (argila) } \\
\hline 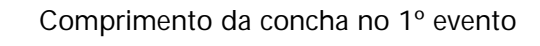 & 11 & 17,0 & 14,12 & 1,42 & 10,090 \\
\hline Comprimento da concha no $2^{\circ}$ evento & 15 & 22,0 & 17,20 & 1,73 & 10,110 \\
\hline Comprimento da concha no 3 o evento & 17 & 23,0 & 19,41 & 1,24 & 6,420 \\
\hline Comprimento da concha no 4 o evento & 19 & 22,5 & 20,57 & 1,10 & 5,363 \\
\hline Comprimento da concha no 5 o evento & 19 & 23,0 & 20,65 & 1,10 & 5,350 \\
\hline \multicolumn{6}{|l|}{ Caixa 3 (terra vegetal) } \\
\hline Comprimento da concha no $1^{\circ}$ evento & 8 & 14,0 & 11,46 & 1,37 & 12,030 \\
\hline Comprimento da concha no $2 \stackrel{\circ}{\circ}$ evento & 10 & 17,0 & 13,50 & 1,84 & 13,690 \\
\hline Comprimento da concha no 3 o evento & 12 & 18,5 & 15,04 & 1,87 & 12,470 \\
\hline Comprimento da concha no 4 o evento & 13 & 20,0 & 16,43 & 2,01 & 12,260 \\
\hline
\end{tabular}

Primeiro evento reprodutivo

A análise de variância (ANOVA, $p<0,05$ ), seguida pelo teste deTamhane, mostrou haver diferença significativa ( $\mathrm{F}: 43,28$; sig.: 0,000 ) entre as médias de comprimento da concha dos indivíduos da caixa 1 (areia) e da caixa 3 (terra vegetal) e entre os indivíduos da caixa 2 (argila) e da caixa 3 (terra vegetal), no primeiro evento reprodutivo. Houve correlação entre idade (Tab. III) e comprimento da concha, no primeiro evento reprodutivo dos indivíduos da caixa 1 (areia) ( $p<0,01$; coeficiente de corre- lação: 0,470; sig.: 0,005), da caixa 2 (argila) ( $p<0,01$; coeficiente de correlação: 0,706; sig.: 0,000) e da caixa 3 (terra vegetal) ( $p<0,05$; coeficiente de correlação: 0,407; sig.: 0,035).

BESSA \& ARAújo (1995a) observaram comprimentos de concha de 9,1-13,5 mm ao primeiro aparecimento de ovos em indivíduos da espécie $S$. octona criados em terra vegetal. No presente estudo, foi observado uma amplitude semelhante para os val ores de comprimento da concha ao primeiro aparecimento de ovos nos indivíduos da caixa 3 (terra vegetal): 8-14 mm. 
Tabela III. Idade (dias), durante os eventos reprodutivos de indivíduos de Subulina octona, mantidos em diferentes substratos.

\begin{tabular}{lccccc}
\hline & Mínimo & Máximo & Média & Desvio padrão Coeficiente de variação (\%) \\
\hline Primeiro evento reprodutivo & & & & & \\
Caixa1 (areia) & 45 & 65 & 50,47 & 5,06 & 10,03 \\
Caixa 2 (argila) & 45 & 69 & 52,81 & 7,08 & 13,41 \\
Caixa 3 (terra vegetal) & 48 & 69 & 59,17 & 7,32 & 12,38 \\
Segundo evento reprodutivo & & & & \\
Caixa1 (areia) & 51 & 84 & 67,54 & 11,92 \\
Caixa 2 (argila) & 61 & 119 & 73,32 & 11,01 & 12,61 \\
Caixa 3 (terra vegetal) & 69 & 105 & 87,25 & 10,47 & 12,00 \\
Terceiro evento reprodutivo & & & & 13,01 & 14,89 \\
Caixa1 (areia) & 64 & 119 & 87,37 & 14,85 \\
Caixa 2 (argila) & 74 & 118 & 89,62 & 13,31 & 9,21 \\
Caixa 3 (terra vegetal) & 84 & 119 & 106,39 & 9,80 & 10,27 \\
Quarto evento reprodutivo & & & & 7,31 \\
Caixa1 (areia) & 74 & 119 & 102,38 & 10,51 & 3,29 \\
Caixa 2 (argila) & 95 & 122 & 110,54 & 8,08 & 3,88 \\
Caixa 3 (terra vegetal) & 105 & 119 & 117,92 & &
\end{tabular}

Os indivíduos das caixas 1 (areia) e 2 (argila), apresentaram comprimentos de concha de $13-16 \mathrm{~mm}$ e de $11-17 \mathrm{~mm}$ respectivamente, ao primeiro aparecimento de ovos, valores mais altos que aqueles verificados por BESSA \& ARAÚJO (1995a). Esses dados evidenciam, mais uma vez, que os substratos areia e argila são mais favoráveis ao crescimento de $S$. octona, sob condições experimentais em que os moluscos são alimentados com ração. BESSA \& ARAÚJO (1995c) observaram que moluscos da espécie $S$. octona alimentados apenas com alface (Lactuca sativa L.), cresceram menos e levaram mais tempo para atingir a maturidade sexual, do que moluscos alimentados com alface e ração para pintos de corte enriquecida com carbonato de cálcio. Resultados semelhantes foram obtidos por MAnsur \& MACHADO (1994) ao submeterem lesmas da espécie $S$. linguaeformis a três diferentes dietas. As lesmas alimentadas com ração mais alface tiveram ganho de peso superior àquelas criadas apenas com ração ou alface.

\section{Segundo evento reprodutivo}

Os indivíduos das caixas 1 (areia) e 2 (argila), apresentaram maior comprimento de concha no segundo evento reprodutivo do que os indivíduos da caixa 3 (terra vegetal). A diferença entre as médias de comprimento da concha foi demonstrada, entre os indivíduos das caixas 1 e 3 e das caixas 2 e 3 (F:46,76; sig.: 0,000). Houve correlação entre a idade (Tab. III) e comprimento da concha dos indivíduos da caixa 1 ( $\mathrm{p}<$ 0,05; sig.: 0,019; coeficiente de correlação: 0,407). Não houve correlação entre idade e comprimento da concha dos indivíduos das caixas 2 e 3.

\section{Terceiro evento reprodutivo}

Os indivíduos da caixa 3 (terra vegetal) apresentaram, no terceiro evento reprodutivo, menor comprimento de concha quando comparados àqueles das caixas 1 (areia) e 2 (argila). A análise de variância (ANOVA, p $<0,05$ ), seguida por teste de Scheffe, mostrou ser significativa a diferença entre as médias de comprimento da concha dos indivíduos das caixas 1 e 3 e das caixas 2 e 3 (F: 88,41; sig.: 0,000). Houve correlação entre a idade (Tab. III) e o comprimento da concha dos indivíduos da caixa 1 (teste de Spearman, $\mathrm{p}<0,001$; coeficiente de correlação =0,638; sig.: 0,000).

\section{Quarto evento reprodutivo}

A análise de variância (ANOVA, $\mathrm{p}<0,05$ ), seguida pelo teste de Tamhane, mostrou ser significativa a diferença entre as médias de comprimento da concha dos indivíduos das caixas 1 e 3 e das caixas 2 e 3 (F:48,88; sig.: 0,000). Não houve correlação entre idade (Tab. III) e comprimento da concha para nenhum dos tratamentos.

\section{Aumento do comprimento da concha de um evento reprodutivo para o subseqüente}

Foi observada uma tendência para a desaceleração do crescimento dos indivíduos submetidos aos três tratamentos (Tab. IV). O comprimento da concha tendeu a aumentar, mas esse aumento tornou-se menos expressivo com a sucessão dos eventos reprodutivos.

A análise de variância (ANOVA, p $<0,05$ ), seguida pelo teste de Tamhane, mostrou haver diferença significativa entre 
Tabela IV. Aumento do comprimento da concha $(\mathrm{mm})$, de um evento reprodutivo para o subseqüente, em indivíduos de Subulina octona, mantidos em diferentes substratos, por 120 dias.

\begin{tabular}{|c|c|c|c|c|c|}
\hline & Mínimo & Máximo & Média & Desvio padrão & $\begin{array}{c}\text { Coeficiente de } \\
\text { variação (\%) }\end{array}$ \\
\hline \multicolumn{6}{|c|}{ Aumento do comprimento da concha do evento 1 para o evento 2} \\
\hline Caixa 1 (areia) & 1,0 & 6,5 & 3,29 & 1,34 & 40,70 \\
\hline Caixa 2 (argila) & 0 & 8,0 & 3,30 & 2,25 & 68,10 \\
\hline Caixa 3 (terra vegetal) & 0 & 5,0 & 1,98 & 1,33 & 67,10 \\
\hline \multicolumn{6}{|c|}{ Aumento do comprimento da concha do evento 2 para o evento 3} \\
\hline Caixa 1 (areia) & 0,5 & 5,5 & 2,80 & 1,28 & 45,71 \\
\hline Caixa 2 (argila) & 0 & 3,5 & 2,19 & 0,99 & 45,20 \\
\hline Caixa 3 (terra vegetal) & 0 & 4,0 & 1,68 & 0,85 & 50,50 \\
\hline \multicolumn{6}{|c|}{ Aumento do comprimento da concha do evento 3 para o evento 4} \\
\hline Caixa 1 (areia) & 0 & 2,0 & 0,94 & 0,48 & 51,00 \\
\hline Caixa 2 (argila) & 0 & 2,5 & 1,12 & 0,76 & 67,80 \\
\hline Caixa 3 (terra vegetal) & 0 & 3,0 & 1,36 & 0,89 & 65,40 \\
\hline
\end{tabular}

as médias de aumento do comprimento da concha, do evento 1 para o evento 2, dos indivíduos da caixa 1 (areia) e 3 (terra vegetal) e 2 (argila) e 3 (terra vegetal) (diferença entre as médias: $-1,23$ e -1,24; sig.: 0,02 e 0,49, respectivamente). Houve diferença significativa também entre as médias de aumento do comprimento da concha, do evento 2 para o evento 3, dos indivíduos das caixas 1 e 3 (diferença entre as médias: -1,11; sig.: 0,02).

O crescimento determinado é o padrão de distribuição de recursos entre o crescimento somático e a reprodução mais observado na natureza. Após o nascimento, toda energia excedente é direcionada para o crescimento, até que a reprodução se inicie. Após o alcance da maturidade, o crescimento cessa e toda a energia excedente é direcionada para a reprodução. Diferentemente, no crescimento indeterminado, o crescimento continua após a maturidade, durante toda a vida do organismo, ou se desacelera após a maturidade e finalmente pára (CiCON 1999). Os resultados deste estudo mostram que o crescimento indeterminado, com o declínio progressivo do crescimento após a maturidade, é a estratégia exibida por $S$. octona. Resultados semelhantes foram obtidos por BESSA \& ARAúJo (1995a), que verificaram um decréscimo na taxa de crescimento de $S$. octona após o alcance da maturidade sexual. Nos primeiros 45 dias do experimento, os autores verificaram um aumento do comprimento da concha dos moluscos em torno de 0,8-1,5 mm e nos últimos dias entre 0,2 e 1,0 mm. Bradybaena similaris (Férussac, 1821) (Xanthonychidae) e Leptinaria unilamellata (d'Orbigny, 1835) (Subulinidae) também apresentaram uma diminuição do ritmo de crescimento no período correspondente à maturidade (Almeida \& Bessa 2001a, b), assim como Achatina (Achatina) achatina (Linné) (Achatinidae) (HodAsI 1979, 1982) e Lymnaea columella (Say, 1817) (Lymnaeidae) (Souza \& MagalHães 2000, GuTIERREz et al. 2001).
Esse padrão de crescimento parece ser ótimo quando a produtividade aumenta com o tamanho do corpo. Neste contexto, o crescimento pós-maturidade pode não ser tão prejudicial ao sucesso reprodutivo desde que os indivíduos compensem a sua baixa produção de filhotes em um dado momento, através de uma maior produtividade futura, garantida por um tamanho de corpo maior (Cicon 1999, Heller 2001). Possivelmente, entre os moluscos pulmonados, este aumento da produtividade com o tempo relaciona-se com o crescimento dos órgãos do aparelho reprodutor e maturação do ovotestis. Durante o crescimento pós-maturidade de Biomphalaria tenagophila (Orbigny) (Planorbiidae), há um alongamento do ovotestis, com aumento do número de folículos, um alongamento da próstata e da bainha do pênis, bem como um aumento do comprimento da glândula muscípara-ootecal (MonteIro \& KawANO 1998). Furtado et al. (2002) observaram que o tempo mínimo e máximo para a ovipostura em indivíduos da espécie $B$. similaris, mantidos agrupados e isolados, foi 94 e 173 dias, respectivamente. Através do exame histológico do ovotestis, foi verificado o primeiro aparecimento de oócitos e espermatozóides aos 70 dias de vida, mas o ovotestis continuou a se diferenciar e a partir do $90^{\circ}$ dia de vida até o $180^{\circ}$ dia, a presença de oócitos tornou-se mais expressiva.

No presente estudo foi observado o aumento da média do comprimento da concha de $S$. octona, com a sucessão dos eventos reprodutivos. Existiu correlação positiva entre o número de ovos produzidos e o comprimento da concha dos indivíduos criados em caixas com areia, no terceiro evento reprodutivo ( $\mathrm{p}<0,05$; coeficiente de correlação: 0,404; sig.: $0,030)$, e dos indivíduos criados em terra vegetal, no primeiro ( $p<0,05$; coeficiente de correlação: 0,411; sig.: 0,033), no terceiro ( $<<0,01$; coeficiente de correlação: 0,619; sig.: 0,001) e 
no quarto evento reprodutivo ( $\mathrm{p}<0,001$; coeficiente de correlação: 0,680; sig.: 0,005). Ou seja, indivíduos maiores produziram maior número de ovos. Estes resultados foram confirmados, no presente estudo, ao se verificar a existência de correlação entre o peso, o comprimento da concha e o número de ovos produzidos por 60 indivíduos, de diferentes idades, da espécie $S$. octona provenientes das criações matrizes. Houve correlação altamente significativa entre peso e comprimento da concha (coeficiente de correlação: 0,841; sig.:0,000), entre peso e número de ovos (coeficiente de correlação: 0,953; sig.: $0,000)$ e entre comprimento da concha e número de ovos (coeficiente de correlação: 0,787; sig.: 0,000). Амato \& Rosa (1982) também verificaram a existência de correlação positiva entre o tamanho de caramujos da espécie Lymnaea (Pseudosuccinea) columella Say e o número médio de ovos produzidos por massa ovígera.

Além da tendência à desaceleração do crescimento foi observada, nos três tratamentos, uma tendência para uma maior homogeneidade (menor variação em relação à média) no comprimento da concha, com o passar do tempo e a sucessão dos eventos reprodutivos. Essa tendência é evidenciada pela diminuição progressiva dos coeficientes de variação.

BESSA \& ARAÚJo (1995a) obtiveram resultados semelhantes. Em suas observações sobre o crescimento de 32 indivíduos da espécie $S$. octona, mantidos isolados por 120 dias, após o aparecimento do primeiro indivíduo com ovo, registraram uma diminuição progressiva do coeficiente de variação da altura da concha de 11,39\% para 6,01\%. Almeida \& BesSA (2001a, b) registraram um decréscimo progressivo da variação, em relação à média, da altura da concha de indivíduos da espécie $B$. similaris, mantidos isolados e L. unilamellata mantidos agrupados. O coeficiente de variação da altura da concha de B. similaris diminuiu de 44,53\%, aos 45 dias de vida, para 12,83\% aos 180 dias de vida, e de L. unilamellata de $12,11 \%$, aos 45 dias de vida, para 4,9\% aos 180 dias. Esta tendência não foi muito evidente para indivíduos da espécie L. unilamellata mantidos isolados. O coeficiente de variação diminuiu até o $90^{\circ}$ dia de vida, voltando a aumentar até o $180^{\circ}$ dia.

\section{CONSIDERAÇÕES FINAIS}

Sob condições experimentais, o substrato influenciou o crescimento de Subulina octona.

A ingestão de substrato faz parte do repertório comportamental dessa espécie, sendo provável que as diferenças no crescimento entre os moluscos criados em terra vegetal e aqueles criados em areia e argila tenham ocorrido em função de um maior consumo de substrato em detrimento do consumo de ração por aqueles moluscos.

O crescimento indeterminado, com o declínio progressivo do aumento do comprimento da concha, após a maturidade é a estratégia exibida por $S$. octona.

Existe correlação entre o comprimento da concha, o peso corporal e o número de ovos produzidos por $S$. octona.

\section{REFERÊNCIAS BIBLIOGRÁFICAS}

Almeida, M.N. \& E.C.A. Bessa. 2001a. Estudo do crescimento e da reprodução de Leptinaria unilamellata (D'Orbigny) (Mollusca, Subulinidae) em laboratório. Revista Brasileira de Zoologia, Curitiba, 18 (4): 1107-1113.

Almeida, M.N. \& E.C.A. Bessa. 2001b. Estudo do crescimento e da reprodução de Bradybaena similaris (Mollusca, Xanthonychidae) em laboratório. Revista Brasileira de Zoologia, Curitiba, 18 (4): 1115-1122.

Aмato, S.B. \& V.L.M. Rosa 1982. Observações sobre a biologia de Lymnaea (Pseudosussinea) columella Say. Número médio de ovos por massa ovígera relacionado ao tamanho do caramujo. Ciência e Cultura, São Paulo, 34 (12): 1640-1642.

Araújo, J.L.B. \& E.C.A. Bessa. 1993. Moluscos de importância econômica do Brasil. II Subulinidae, Subulina octona (Brugüière) (Mollusca, Gastropoda, Pulmonata, Stylommatophora). Revista Brasileira de Zoologia, Curitiba, 10 (3): 489-497.

Assami, T. \& К. ОвауASHI. 1999. Effects of oviposition substrate on lifetime fecundity of terrestrial pulmonate Bradybaena similaris. Journal of Conchology, Ireland, 36 (5): 3-9.

Bessa, E.C.A. \& J.L.B. Araújo. 1995a. Oviposição, tamanho de ovos e medida do comprimento da concha em diferentes fases do desenvolvimento de Subulina octona (Brugüière) (Pulmonata, Subulinidae) em condições de laboratório. Revista Brasileira de Zoologia, Curitiba, 12 (3): 647-654.

Bessa, E.C.A. \& J.L.B. ARAúJo. 1995b. Ocorrência de autofecundação em Subulina octona (Brugüière) (Pulmonata, Subulinidae) em condições de laboratório. Revista Brasileira de Zoologia, Curitiba, 12 (3): 719-723.

Bessa, E.C.A. \& J.L.B. Araújo. 1995c. Influência da alimentação com ração concentrada no desenvolvimento de Subulina octona Brugüière, 1789 (Mollusca: Subulinidae) em condições de laboratório. Boletim do Instituto de Ciências Biológicas, Juiz de Fora, 47: 21-27.

Chatfield, J.E. 1973. Aspects of feeding and growth in land snails. Malacologia, Ann Arbor, 14: 391-392.

Chatfield, J.E. 1976. Studies on food and feeding in some european land molluscs. Journal of Conchology, Ireland, 29: 5-20.

Chevalier, L.; C. Desbuquois; J. Lelannic \& M. Charrier. 2001. Poace in the natural diet of the snail Helix aspersa Muller (Gastropoda, Pulmonata). Life Sciences, Paris, 324: 979-987.

Cicon, M. 1999. Growth after maturity as a suboptimal strategy. Acta Oecologica, Paris, 20 (1): 25-28.

ClampitT, P.T. 1973. Substratum as a factor in the distribution of pulmonate snails in Douglas Lake, Michigan. Malacologia, Ann Arbor, 12 (2): 379-399.

Соoк, A. 1985. Functinal aspects of trail following by the carnivorous snail Euglandina rosea. Malacologia, Ann Arbor, 26 (1-2): 173-181.

D'ÁVILA, S. \& E.C.A. BESSA. 2005. Influência do substrato sobre a reprodução de Subulina octona (Brugüière) (Mollusca, Subulinidae), sob condições de laboratório. Revista Brasileira 
de Zoologia, Curitiba, 22 (1): 197-204.

Dutra, A.V.C. 1980. Aspectos da ecologia e da reprodução de Leptinaria unilamellata (Orbigny, 1835) (Gastropoda, Subulinidae). Revista Brasileira de Zoologia, Curitiba, 5 (4): 581-591.

Elwell, A.S. \& M.J. Ulmer. 1971. Notes on the biology of Anguispira alternata (Stylommatophora: Endodontidae). Malacologia, Ann Arbor, 11 (1): 199-216.

Fisher, P.H. \& J.C.H. Crosse. 1878. Mission Scientifique au Mexique et dans l'Amerique Centrale. Recherches Zoologiques, Paris, 7 (l): 1-731.

Furtado, M.C.V.; E.C.A. Bessa \& M.C.M. Castañon. 2002. Hystological characterization of ovotestis of Bradybaena similaris (Férussac,1821) (Mollusca, Xanthonychidae) in different developmental phases, kept isolated or in groups, under laboratorial conditions. Revista Brasileira de Zoociências, Juiz de Fora, 4 (2): 229-300.

Gutiérrez, A.; G. Perera; M. Yong \& L. Wong. 2001. The effect of isolation on the life-story traits of Pseudosuccinea columella (Pulmonata: Lymnaeidae). Memórias do Instituto Oswaldo Cruz, Rio de janeiro, 96 (4): 577-581.

HeLler, J. 2001. Life story strategies, p. 413-445. In: G.M. BARKer (Ed.). The biology of terrestrial molluscs. New Zeland, CABI publishing, 558p.

HoDASI, J.K.M. 1979. Life story studies of Achatina (Achatina) achatina (Linné). Journal of Molluscan Studies, Londres, 45: 328-339.

HodASI, J.K.M. 1982. The effects of different ligth regimes on the behaviour and biology of Achatina (Achatina) achatina (Linné). Journal of Molluscan Studies, Londres, 48: 283-293.

Mansur, G.G. \& M.P. Machado. 1994. Nota preliminar sobre três tipos de dietas para a alimentação de Sarassinula linguaeformis (Semper, 1885) (Gastropoda, Veronicellidae) em condições de laboratório. Biociências, Porto Alegre, 2 (1): 71 74.

Monteiro, W. \& T. KaWano. 1998. Crescimento de órgãos do aparelho reprodutor durante o desenvolvimento de Biomphalaria tenagophila (Orbigny) (Mollusca, Planorbidae). Revista Brasileira de Biologia, Rio de Janeiro, 58 (4): 693-705.

Ninei, N.; S. Asami \& H. TanaKa. 1981. Geographical factors influencing the population numbers and distribution of Oncomelania nosophora and the subsequent effect on the control of shistosomiasis japonica in Japan. Social Science and Medicine, Glasgow, 15: 149-157.

Ondina, P.; S. Mato; J. Hermina \& A. Outeiro. 1998. Importance of soil exchangeable cations and aluminium content on land snail distribution. Applied Soil Ecology, Ohio, 9: 229-232.

Outeiro, A.; P. Ondina; T. Rodrigues \& J. Castillejo. 1989. Autoecological study of Punctum (P.) pygmaeum (Drad., 1801) (Gastropoda, Pulmonata) in the Sierra de O. Cowel, Lugo, Spain. Revue d' Écologie et de Biologie du Sol, Paris, 26 (4): 515-525.

SouZA, C.P. \& K.G. MAgalhães. 2000. Rearing of Lymnaea columella (Say, 1917), intermediate host of Fasciola hepatica (Linnaeus, 1758). Memórias do Instituto Oswaldo Cruz, Rio de Janeiro, 95 (5): 739-741.

Speiser, B. 2001. Food and feeding behaviour, p. 259-2888. In: G. M. BARKER (Ed.). The Biology of terrestrial molluscs. New Zeland, CABI Publishing, 558p.

Voss, M.; A. Utech \& W. WÜnnemberg. 2001. The dependence of thermopreferendum in Helix pomatia L. on air temperature. Journal of Thermal Biology, Durhan, 26: 155-158.

Recebido em 20.IV.2004; aceito em 17.II.2005. 